\title{
Prehospital pulse oximetry: a red flag for early detection of silent hypoxemia in COVID-19 patients
}

\author{
Romain Jouffroy, Daniel Jost ${ }^{*}$ (D) and Bertrand Prunet
}

The COVID-19 pandemic, which has been expanding since the first cases in Asia in late 2019, may result in acute respiratory failure (ARF) with severe hypoxemia [1-3]. In prehospital settings, the paucity of clinical respiratory signs has made assessing the severity of some COVID-19 patients challenging. Indeed, even though hypoxic ARF generally leads to an increase in respiratory rate (RR) [4], in some COVID-19 patients, a persistent normal RR was inconsistent with the severity of hypoxia.

Based on retrospective data, we aimed to describe the discrepancy between prehospital initial RR (RRi) and initial $\mathrm{SpO} 2$ (Spo2i; i.e., before oxygen supplementation, $\mathrm{FiO} 2=21 \%$ ) in COVID-19 patients suffering from ARF.

We retrospectively examined consecutive COVID-19 patients suffering from ARF who were treated by the Paris Fire Brigade's basic life-support (BLS) teams in the prehospital setting. Data were provided from primary home care providers. Based on a previous study [5], we used the $\mathrm{SpO} 2 \mathrm{i} / \mathrm{RR}$ ratio as an estimator of the discrepancy insofar as a low numerator is associated with hypoxia, whereas a high denominator is typically associated with respiratory failure.

After having measured the $\mathrm{SpO} 2 \mathrm{i} / \mathrm{RRi}$ values in COVID-19 patients, we compared them to those of nonCOVID-19 patients (i.e., patients with other causes of ARF treated by the BLS teams over the previous 3 years in the same period).

Continuous data were described as median (interquartile range) and were compared by applying the KruskalWallis test. The French Society of Anaesthesia and
Intensive Care approved the trial protocol on April 7, 2020 (IRB 00010254-2020-055).

The study examined 1201 patients who experienced COVID-19 between March 13 and 29, 2020. The median $\mathrm{SpO} 2 \mathrm{i} / \mathrm{RR}$ value was significantly higher than that of patients treated in the previous 3 years $(5[4,5]$ in 2020 versus 3.4 [2.4-4.5] in 2019, 3.3[2.2-4.4] in 2018, and $3.5[2.5-4.6]$ in 2017, $p<0.001$, Fig. 1).

In summary, this retrospective study based on prehospital first responder data highlighted a relatively higher discrepancy between SpO2i and RRi in COVID-19 ARF patients, in comparison with previous non-COVID-19 ARF patients. Without a systematic SpO2i measurement, a normal breathing rate could mask profound hypoxia and make severity assessment in COVID-19 patients all the more difficult in an out-of-hospital setting.

Despite differences in worldwide prehospital emergency medical services, pulse oximetry is an accessible tool that prehospital healthcare providers can easily use.

In conclusion, prehospital pulse oximetry might be used as a red flag for early detection of "silent hypoxemia" in COVID-19 patients. The prehospital SpO2i/RRi ratio needs further investigation because it might help to identify non-clinically obvious ARFs.

\footnotetext{
* Correspondence: Daniel.jost@pompiersparis.fr

Paris Fire Brigade, Emergency Medicine Department, 1 Place Jules Renard, 75017 Paris, France
} 

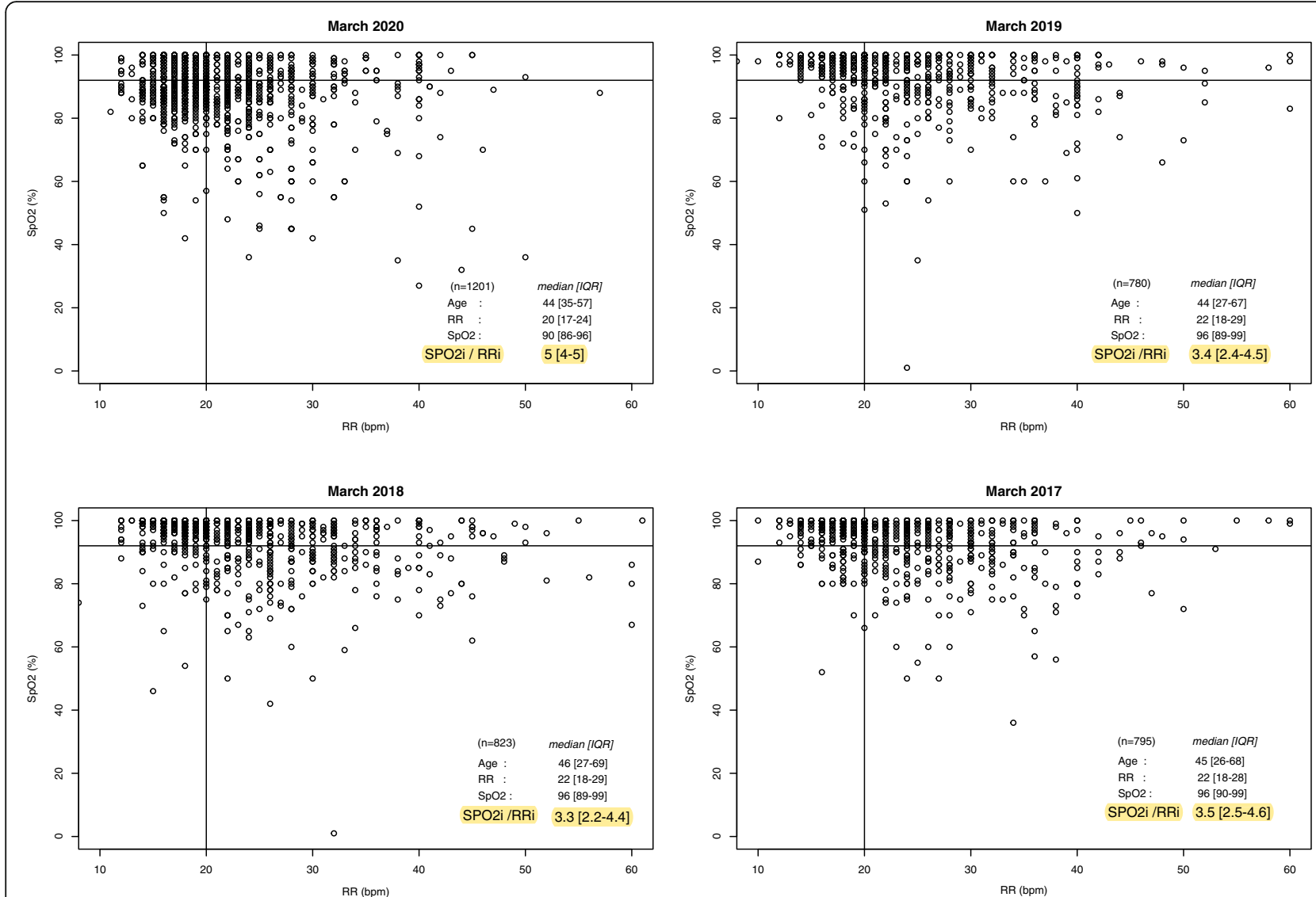

Fig. 1 Scatter plot representing the initial SPO2 (SPO2i) and initial respiration rate (RRi) values for each patient, for COVID-19 (March 2020) and non-COVID-19 patients from the previous 3 years. The horizontal and vertical lines indicate the threshold values of SPO2 95\% and respiration rate, 20 breaths per minute, respectively. We used the Kruskal-Wallis test to compare the median SPO2i/RRi value, between the period " $13^{\text {th }}$ to March 29,2020 ," and the three previous years ( $p$ value $<0.001$ ). RRi, initial respiratory rate; SpO2i, initial pulse oximetry value; $N$, number of patients included

\section{Acknowledgements}

Thanks to the Paris Fire Brigade Basic Life Support Teams for their daily engagement during the pandemic.

\section{Authors' contributions}

All authors have made substantial contributions to the conception and design of the letter, drafting the article or revising it critically for relevant intellectual content, and final approval of the version to be submitted. The authors read and approved the final manuscript

\section{Funding}

None

\section{Availability of data and materials}

The datasets used and/or analyzed during the current study are available from the corresponding author on reasonable request.

\section{Ethics approval and consent to participate}

As a retrospective study design, the study protocol has been submitted to the Ethics Committee of "The French Society of Anaesthesia and Intensive Care." The trial protocol was approved on April 7, 2020 (IRB 00010254-2020055).

\section{Consent for publication}

Not applicable

\section{Competing interests}

The authors declare that they have no competing interests.

Received: 20 May 2020 Accepted: 27 May 2020

Published online: 08 June 2020

\section{References}

1. Verity R, Okell LC, Dorigatti I, et al. Estimates of the severity of coronavirus disease 2019: a model-based analysis. Lancet Infect Dis. 2020;S14733099(20):30243-7.

2. Onder G, Rezza G, Brusaferro S. Case-fatality rate and characteristics of patients dying in relation to COVID-19 in Italy. JAMA. 2020. https://doi.org/ 10.1001/jama.2020.4683 Accessed on May 15, 2020.

3. Guan WJ, Ni ZY, Hu Y, et al. Clinical characteristics of coronavirus disease 2019 in China. N Engl J Med. 2020:382:1708-20.

4. Braman SS. The regulation of normal lung function. Allergy Asthma Proc. 1995:16:223-6.

5. Roca O, Messika J, Caralt B, et al. Predicting success of high-flow nasal cannula in pneumonia patients with hypoxemic respiratory failure: the utility of the ROX index. J Crit Care. 2016;35:200-5.

\section{Publisher's Note}

Springer Nature remains neutral with regard to jurisdictional claims in published maps and institutional affiliations. 